\title{
ON ESTIMATION OF THE VARIANCES FOR CRITICAL BRANCHING PROCESSES WITH IMMIGRATION
}

\author{
CHUNHUA MA ${ }^{* * *}$ AND \\ LONGMIN WANG, ${ }^{* * * *}$ Nankai University
}

\begin{abstract}
The conditional least-squares estimators of the variances are studied for a critical branching process with immigration that allows the offspring distributions to have infinite fourth moments. We derive different forms of limiting distributions for these estimators when the offspring distributions have regularly varying tails with index $\alpha$. In particular, in the case in which $2<\alpha<\frac{8}{3}$, the normalizing factor of the estimator for the offspring variance is smaller than $\sqrt{n}$, which is different from that of Winnicki (1991).
\end{abstract}

Keywords: Branching process with immigration; regular variation; conditional least squares estimator; limiting distribution

2010 Mathematics Subject Classification: Primary 60J35

Secondary 60J80; 60H20; 60K37

\section{Introduction}

Let $\left\{\xi_{k, j}: k, j=1,2, \ldots\right\}$ and $\left\{\eta_{k}: k=1,2, \ldots\right\}$ be two independent families of independent and identically distributed (i.i.d.) random variables taking values in $\mathbb{N}:=\{0,1,2, \ldots\}$. A Galton-Watson branching process with immigration (GWI process) $\left\{X_{k}: k=1,2, \ldots\right\}$ is defined inductively by

$$
X_{0}=0, \quad X_{k}=\sum_{j=1}^{X_{k-1}} \xi_{k, j}+\eta_{k}, \quad k \geq 1 .
$$

Intuitively, the distribution of $\xi_{k, j}$ is called the offspring distribution and the distribution of $\eta_{k}$ is called the immigration distribution. Let $g(\cdot)$ and $h(\cdot)$ be the generating functions of $\xi_{k, j}$ and $\eta_{k}$, respectively. It is easy to see that $\left\{X_{k}\right\}$ is a discrete-time Markov chain with values in $\mathbb{N}$ and one-step transition matrix $P(i, j)$ given by

$$
\sum_{j=0}^{\infty} P(i, j) s^{j}=g(s)^{i} h(s), \quad i \in \mathbb{N}, 0 \leq s \leq 1 .
$$

Let $m=\mathrm{E}\left[\xi_{k, j}\right], \sigma^{2}=\operatorname{var}\left[\xi_{k, j}\right], \lambda=\mathrm{E}\left[\eta_{k}\right]$, and $\gamma^{2}=\operatorname{var}\left[\eta_{k}\right]$. The cases $m>1, m=1$, and $m<1$ are respectively referred to as supercritical, critical, and subcritical.

Received 4 November 2009; revision received 6 February 2010.

* Postal address: School of Mathematical Sciences and LPMC, Nankai University, Tianjin 300071, P. R. China.

Supported by the NSFC (grant numbers 10871103 and 10971106).

** Email address: mach@nankai.edu.cn

*** Email address: wanglm@nankai.edu.cn 
The estimation problem for the variances $\sigma^{2}$ and $\gamma^{2}$ in the GWI process has been extensively studied; see [7], [18], and the references therein. A natural method is the conditional leastsquares estimator (CLSE) in the sense of Klimko and Nelson [11] or Winnicki [18]. Winnicki also examined the asymptotic properties of the estimators for the variances under the conditions that $\mathrm{E}\left[\xi_{1,1}^{4}\right]<\infty$ and $\mathrm{E}\left[\eta_{1}^{4}\right]<\infty$. He showed that the CLSE of the offspring variance is consistent only if $m \leq 1$. In the subcritical case, the CLSE is asymptotically normal with the normalizing factors $n^{1 / 2}$, while in the critical case the CLSE is not asymptotically normal but it has another limit law with the normalizing factor $n^{1 / 2}$. The limit law is expressed in terms of a Brownian motion and the limit process resulting from the weak convergence of rescaled GWI processes.

In this paper we consider a similar estimation problem in a critical GWI process, and derive the asymptotic distributions of the estimators without assuming that $\mathrm{E}\left[\xi_{1,1}^{4}\right]<\infty$ and $\mathrm{E}\left[\eta_{1}^{4}\right]<\infty$. We restrict our attention to the critical case, since in this case the asymptotic behavior of the CLSE is closely related to some limit theorems of the GWI processes, and is of special interest in considering the limit theorems in a heavy-tailed setting allowing finite variances but infinite fourth moments. Throughout the paper, we assume that the $\left\{\xi_{k, j}\right\}$ have regularly varying tails. Specifically,

$$
\mathrm{P}\left(\xi_{1,1}>x\right) \sim x^{-\alpha} L(x) \text { as } x \rightarrow \infty \text { for } \alpha>2,
$$

where $L(x)$ is a positive function slowly varying at $\infty$. Note that if $2<\alpha<4, \mathrm{E}\left[\xi_{1,1}^{4}\right]=\infty$ and $\mathrm{E}\left[\xi_{1,1}^{2+\delta}\right]<\infty$ for $0<\delta<\alpha-2$. We refer the reader to [3, pp. 330-337] for a systematic study of distributions with regularly varying tails.

Our main result (Theorem 2.2) shows that if $2<\alpha<\frac{8}{3}$, the CLSE of the offspring variance has a limit law with normalizing factor smaller than $n^{1 / 2}$, and if $\alpha>\frac{8}{3}$, the normalizing factor is $n^{1 / 2}$. When $\alpha=\frac{8}{3}$, the normalizing factor depends on the behavior of the slowly varying part $L(\cdot)$. It is also interesting to note that, when $2<\alpha \leq \frac{8}{3}$, the form of the limit law for the CLSE may involve an $(\alpha / 2)$-stable process and the limit process of the rescaled GWI processes. This is different from that of [18].

The remainder of this paper is organized as follows. In Section 2 we give the main limit theorem, and then the asymptotic estimates for the variances of the GWI process as an application of our limit theorem. Section 3 is devoted to the proofs of the above results.

Notation. Let $\mathbb{R}_{+}=[0, \infty)$. We respectively denote by ' $\stackrel{\mathrm{P}}{\rightarrow}$ ' and ' $\rightarrow$ ' the convergence of random variables in probability and convergence in distribution, we denote by ' $\stackrel{\mathrm{W}}{\rightarrow}$ ' the weak convergence in Skorokhod space. We also use the convention that $\int_{r}^{t}=-\int_{t}^{r}=\int_{(r, t]}$ and $\int_{r}^{\infty}=\int_{(r, \infty)}$ for $r \leq t \in \mathbb{R}$.

\section{Estimators and limit theorems}

Consider the GWI process given in (1.1). For $k \geq 0$, let $\mathcal{F}_{k}$ denote the $\sigma$-algebra generated by $\left\{X_{j}: j=0,1, \ldots, k\right\}$. Let $U_{k}=X_{k}-m X_{k-1}-\lambda$, and let $V_{k}=U_{k}^{2}-\sigma^{2} X_{k-1}-\gamma^{2}$. Then $V_{k}$ is a martingale difference with respect to $\mathcal{F}_{k}$. We treat

$$
U_{k}^{2}=\sigma^{2} X_{k-1}+\gamma^{2}+V_{k}, \quad k=1,2, \ldots, n,
$$

as a stochastic regression equation with unknown coefficients $\sigma^{2}$ and $\gamma^{2}$ and an 'error' term $V_{k}$. If the means $m$ and $\lambda$ are known, the $\operatorname{CLSE}\left(\hat{\sigma}_{n}^{2}, \hat{\gamma}_{n}^{2}\right)$ of $\left(\sigma^{2}, \gamma^{2}\right)$ resulting from (2.1) is

$$
\hat{\sigma}_{n}^{2}=\frac{\sum_{k=1}^{n} U_{k}^{2}\left(X_{k-1}-\bar{X}_{n}^{*}\right)}{\sum_{k=1}^{n}\left(X_{k-1}-\bar{X}_{n}^{*}\right)^{2}}, \quad \hat{\gamma}_{n}^{2}=\sum_{k=1}^{n} \frac{U_{k}^{2}}{n}-\hat{\sigma}_{n}^{2} \bar{X}_{n}^{*},
$$


where $\bar{X}_{n}^{*}=(1 / n) \sum_{k=1}^{n} X_{k-1}$. If $m$ and $\lambda$ are unknown, we get $\left(\bar{\sigma}_{n}^{2}, \bar{\gamma}_{n}^{2}\right)$ by using $\hat{U}_{k}=$ $X_{k}-\hat{m}_{n} X_{k-1}-\hat{\lambda}_{n}$ instead of $U_{k}$ in (2.2), where $\left(\hat{m}_{n}, \hat{\lambda}_{n}\right)$ are the CLSE of $(m, \lambda)$ given in [17]. To obtain the asymptotic behavior of $\left(\hat{\sigma}_{n}^{2}, \hat{\gamma}_{n}^{2}\right)$ or $\left(\bar{\sigma}_{n}^{2}, \bar{\gamma}_{n}^{2}\right)$ in the critical case, as in [18], we need to establish some weak convergence results for the processes that allow the offspring distributions to have infinite fourth moments. Now introduce the sequences

$$
Y_{n}(t)=\frac{X([n t])}{n} \quad \text { and } \quad V_{n}(t)=\sum_{k=1}^{[n t]} V_{k}
$$

for $t \geq 0$, where [nt] denotes the integer part of $n t$. Wei and Winnicki [16] gave the following limit theorem for the sequence $Y_{n}(\cdot)$, where the limit process is a continuous-state branching process with immigration (CBI process). See [13] for the result in generality. Also, see [10] for a complete characterization of the class of CBI processes.

Proposition 2.1. ([16].) Suppose that $m=1, \sigma^{2}<\infty$, and $\gamma^{2}<\infty$. Then $Y_{n}(\cdot)$ converges in distribution on $D\left([0, \infty), \mathbb{R}_{+}\right)$to a CBI process defined by

$$
Y(t)=\lambda t+\int_{0}^{t} \sigma \sqrt{Y(s)} \mathrm{d} W(s)
$$

where $W(\cdot)$ is a one-dimensional Brownian motion.

It follows from (1.2) that $\mathrm{P}\left(\xi_{1,1}>x\right) \sim \mathrm{P}\left(\left|\xi_{1,1}-1\right|>x\right)$ as $x \rightarrow \infty$. So we can find a sequence of positive constants $\left\{a_{n}\right\}$ such that

$$
n^{2} \mathrm{P}\left(\left|\xi_{1,1}-1\right|>a_{n}\right) \rightarrow 1 \text { as } n \rightarrow \infty .
$$

Then we have $a_{n} \sim\left(n^{2} L\left(a_{n}\right)\right)^{1 / \alpha}$. In fact, $a_{n}$ may be defined as $\inf \left\{x: \mathrm{P}\left(\left|\xi_{1,1}-1\right|>x\right) \leq\right.$ $\left.n^{-2}\right\}$. In other words, $a_{n}=n^{2 / \alpha} L^{*}(n)$ for some slowly varying function $L^{*}(x)$. Recall that $W(t)$ is a Brownian motion. Let $B(t)$ be another Brownian motion. For $2<\alpha<4$, let $S_{\alpha / 2}(t)$ be a spectrally positive $(\alpha / 2)$-stable Lévy process with exponent

$$
\theta \mapsto \frac{\alpha}{2} \int_{0}^{\infty}\left(\mathrm{e}^{\mathrm{i} \theta u}-1-\mathrm{i} \theta u\right) \frac{1}{u^{\alpha / 2+1}} \mathrm{~d} u .
$$

Suppose that $W(t), B(t)$, and $S_{\alpha / 2}(t)$ are independent of each other. We have the following theorem.

Theorem 2.1. Assume that $m=1, \gamma^{2}<\infty$, and condition (1.2) is satisfied.

(i) If $\alpha \in\left(2, \frac{8}{3}\right)$ or if $\alpha=\frac{8}{3}$ and $L\left(a_{n}\right) \rightarrow \infty$, then $\left(Y_{n}(\cdot), V_{n}(\cdot) / a_{n}^{2}\right) \stackrel{\mathrm{w}}{\rightarrow}(Y(\cdot), V(\cdot))$ on $D\left([0, \infty), \mathbb{R}_{+} \times \mathbb{R}\right)$ as $n \rightarrow \infty$, where $Y(\cdot)$ is defined by $(2.3)$ and $V(\cdot)$ is defined by

$$
V(t)=\int_{0}^{t} Y^{2 / \alpha}(s) \mathrm{d} S_{\alpha / 2}(s)
$$

(ii) If $\alpha=\frac{8}{3}$ and $L\left(a_{n}\right) \sim c$ for some $c>0$, then $\left(Y_{n}(\cdot), V_{n}(\cdot) / n^{3 / 2}\right) \stackrel{\mathrm{w}}{\rightarrow}(Y(\cdot), V(\cdot))$ on $D\left([0, \infty), \mathbb{R}_{+} \times \mathbb{R}\right)$ as $n \rightarrow \infty$, where $Y(\cdot)$ is defined by $(2.3)$ and $V(\cdot)$ is defined by

$$
V(t)=\int_{0}^{t} \sqrt{2} \sigma^{2} Y(s) \mathrm{d} B(s)+\int_{0}^{t}(c Y(s))^{2 / \alpha} \mathrm{d} S_{\alpha / 2}(s) .
$$


(iii) If $\alpha \in\left(\frac{8}{3}, \infty\right)$ or if $\alpha=\frac{8}{3}$ and $L\left(a_{n}\right) \rightarrow 0$, then $\left(Y_{n}(\cdot), V_{n}(\cdot) / n^{3 / 2}\right) \stackrel{\mathrm{w}}{\rightarrow}(Y(\cdot), V(\cdot))$ on $D\left([0, \infty), \mathbb{R}_{+} \times \mathbb{R}\right)$ as $n \rightarrow \infty$, where $Y(\cdot)$ is defined by $(2.3)$ and $V(\cdot)$ is defined by

$$
V(t)=\int_{0}^{t} \sqrt{2} \sigma^{2} Y(s) \mathrm{d} B(s) .
$$

Remark 2.1. Compared with Lemma 2.8 of [18], Theorem 2.1 shows that there might be a heavy-tailed effect on the limit behavior of $V_{n}(\cdot)$ when $\mathrm{E}\left[\xi_{1,1}^{4}\right]=\infty$. In fact, we decompose $V_{n}(\cdot)$ into three parts: $V_{n}(\cdot)=\sum_{j=1}^{3} V_{j, n}(\cdot)$ (see (3.1), below). If $\alpha \in\left(2, \frac{8}{3}\right)$, the limit behavior of $V_{n}(\cdot)$ is governed by $V_{1, n}(\cdot)$, in which $\left(\xi_{k, j}-1\right)^{2}$ is in the domain of attraction of a stable law with exponent $\alpha / 2$. If $\alpha \in\left(\frac{8}{3}, \infty\right)$, the limit behavior of $V_{n}(\cdot)$ is governed by $V_{2, n}(\cdot)$ which follows, in some sense, from the martingale central limit theorem as in [18, Lemma 2.4]. In the case in which $\alpha=\frac{8}{3}$, the behavior of $V_{n}(\cdot)$ involves the 'mixing' effects of $V_{1, n}(\cdot)$ and $V_{2, n}(\cdot)$.

As an application of Theorem 2.1, our main result is as follows.

Theorem 2.2. Suppose that the conditions of Theorem 2.1 are satisfied with $\lambda>0$. Then there exist sequences of positive constants, $\left\{b_{n}\right\}$ and $\left\{c_{n}\right\}$, such that

$$
\left(\begin{array}{c}
b_{n}\left(\hat{\sigma}_{n}^{2}-\sigma^{2}\right) \\
c_{n}\left(\hat{\gamma}_{n}^{2}-\gamma^{2}\right)
\end{array}\right) \stackrel{\mathrm{D}}{\rightarrow}\left(\begin{array}{c}
\frac{\int_{0}^{1} Y(t) \mathrm{d} V(t)-V(1) \int_{0}^{1} Y(t) \mathrm{d} t}{\int_{0}^{1} Y^{2}(t) \mathrm{d} t-\left(\int_{0}^{1} Y(t) \mathrm{d} t\right)^{2}} \\
\frac{V(1) \int_{0}^{1} Y^{2}(t) \mathrm{d} t-\int_{0}^{1} Y(t) \mathrm{d} t \int_{0}^{1} Y(t) \mathrm{d} V(t)}{\int_{0}^{1} Y^{2}(t) \mathrm{d} t-\left(\int_{0}^{1} Y(t) \mathrm{d} t\right)^{2}}
\end{array}\right)
$$

where $Y(\cdot)$ is defined by (2.3), and $b_{n}, c_{n}$, and $V(\cdot)$, depending on the tail index $\alpha$, are given as follows.

(i) In the case of Theorem 2.1(i), $b_{n}=n^{2} / a_{n}^{2}, c_{n}=n / a_{n}^{2}$, and $V(\cdot)$ is defined by (2.6).

(ii) In the case of Theorem 2.1(ii), $b_{n}=\sqrt{n}, c_{n}=1 / \sqrt{n}$, and $V(\cdot)$ is defined by (2.7).

(iii) In the case of Theorem 2.1(iii), $b_{n}=\sqrt{n}, c_{n}=1 / \sqrt{n}$, and $V(\cdot)$ is defined by (2.8).

Furthermore, (2.9) still holds if $\hat{\sigma}_{n}^{2}$ and $\hat{\gamma}_{n}^{2}$ are replaced by $\bar{\sigma}_{n}^{2}$ and $\bar{\gamma}_{n}^{2}$, respectively.

Remark 2.2. Let $N(0,1)$ be a unit normal distribution, and let $S_{\alpha / 2}(1, \beta, 0)$ be an $(\alpha / 2)$-stable distribution with exponent

$$
\theta \mapsto \exp \left(-|\theta|^{\alpha / 2}\left(1-\mathrm{i} \beta(\operatorname{sgn} \theta) \tan \frac{\pi \alpha}{4}\right)\right),
$$

where $\beta \in[-1,1]$, and

$$
\operatorname{sgn} \theta= \begin{cases}1 & \text { if } \theta>0 \\ 0 & \text { if } \theta=0 \\ -1 & \text { if } \theta<0\end{cases}
$$

in the sense of [14, pp. 5 and 9]. Using this notation, the distribution of $S_{\alpha / 2}(t)$ is $\left(\mathcal{A}_{\alpha / 2} t\right)^{2 / \alpha}$ $\times S_{\alpha / 2}(1,1,0)$ for $t>0$, where $\mathcal{A}_{\alpha / 2}=\pi /(2 \Gamma(\alpha / 2) \sin (\pi \alpha / 4))$. By direct calculation we can show that the limiting random variable of $b_{n}\left(\hat{\sigma}_{n}^{2}-\sigma^{2}\right)$ has the distribution of a mixture

$$
\begin{gathered}
\kappa_{1} S_{\alpha / 2}(1,1,0)+\kappa_{2} S_{\alpha / 2}(1,-1,0), \\
c^{2 / \alpha} \kappa_{1} S_{\alpha / 2}(1,1,0)+c^{2 / \alpha} \kappa_{2} S_{\alpha / 2}(1,-1,0)+\kappa_{3} N(0,1), \quad \text { or } \quad \kappa_{3} N(0,1),
\end{gathered}
$$


respectively according to whether the range of $\alpha$ is as in case (i), (ii), or (iii) of Theorem 2.1, where

$$
\begin{aligned}
\kappa_{1} & =\frac{\left(\mathcal{A}_{\alpha / 2} \int_{0}^{1} \mathbf{1}_{[0, \infty)}\left(\left(Y(t)-\int_{0}^{1} Y(s) \mathrm{d} s\right) Y(t)\left(Y(t)-\int_{0}^{1} Y(s) \mathrm{d} s\right)^{\alpha / 2} \mathrm{~d} t\right)^{2 / \alpha}\right.}{\int_{0}^{1} Y^{2}(t) \mathrm{d} t-\left(\int_{0}^{1} Y(t) \mathrm{d} t\right)^{2}}, \\
\kappa_{2} & =\frac{\left(\mathcal{A}_{\alpha / 2} \int_{0}^{1} \mathbf{1}_{(-\infty, 0)}\left(\left(Y(t)-\int_{0}^{1} Y(s) \mathrm{d} s\right) Y(t)\left(\int_{0}^{1} Y(s) \mathrm{d} s-Y(t)\right)^{\alpha / 2} \mathrm{~d} t\right)^{2 / \alpha}\right.}{\int_{0}^{1} Y^{2}(t) \mathrm{d} t-\left(\int_{0}^{1} Y(t) \mathrm{d} t\right)^{2}},
\end{aligned}
$$

and

$$
\kappa_{3}=\frac{\sqrt{2} \sigma^{2}\left(\int_{0}^{1} Y^{2}(t)\left(Y(t)-\int_{0}^{1} Y(s) \mathrm{d} s\right)^{2} \mathrm{~d} t\right)^{1 / 2}}{\int_{0}^{1} Y^{2}(t) \mathrm{d} t-\left(\int_{0}^{1} Y(t) \mathrm{d} t\right)^{2}} .
$$

It is clear that, for any $K \geq 0, \mathrm{P}(Y(s)=K$ for all $s \in[0,1])=0$. Then $\mathrm{P}\left(\kappa_{1}+\kappa_{2}=0\right)=0$, $\mathrm{P}\left(\kappa_{3}=0\right)=0$, and $\hat{\sigma}_{n}$ is consistent. On the other hand, the limiting random variable of $c_{n}\left(\hat{\gamma}_{n}^{2}-\gamma^{2}\right)$ has a similar form and we have $\left|\hat{\gamma}_{n}^{2}-\gamma^{2}\right| \stackrel{\mathrm{P}}{\rightarrow} \infty$, i.e. $\hat{\gamma}_{n}^{2}$ is not a consistent estimator.

\section{Proofs of the main results}

Let $\overline{\mathcal{F}}_{n}$ be the $\sigma$-field generated by $\left\{X_{0}, \xi_{k, j}, \eta_{k}: 1 \leq k \leq n, j \geq 1\right\}$. Recall that $V_{n}(t)=$ $\sum_{k=1}^{[n t]} V_{k}$. By (1.1), $V_{n}(t)$ can be rewritten in the following form:

$$
\begin{aligned}
V_{n}(t)= & \sum_{k=1}^{[n t]} \sum_{j=1}^{X_{k-1}}\left[\left(\xi_{k, j}-1\right)^{2}-\sigma^{2}\right]+2 \sum_{k=1}^{[n t]} \sum_{j=2}^{X_{k-1}} \sum_{l=1}^{j-1}\left(\xi_{k, j}-1\right)\left(\xi_{k, l}-1\right) \\
& +\left(2 \sum_{k=1}^{[n t]} \sum_{j=1}^{X_{k-1}}\left(\xi_{k, j}-1\right)\left(\eta_{k}-\lambda\right)+\sum_{k=1}^{[n t]}\left[\left(\eta_{k}-\lambda\right)^{2}-\gamma^{2}\right]\right) \\
= & V_{1, n}(t)+V_{2, n}(t)+V_{3, n}(t) .
\end{aligned}
$$

To prove Theorem 2.1, it suffices to study the limit behavior of $\left(V_{1, n}(\cdot), V_{2, n}(\cdot), V_{3, n}(\cdot)\right)$. The following lemma tells us that $V_{3, n}(\cdot)$, after rescaling, can be negligible.

Lemma 3.1. Assume that $m=1, \sigma^{2}<\infty$, and $\gamma^{2}<\infty$. For $d>1, V_{3, n}(\cdot) / n^{d} \stackrel{\mathrm{P}}{\rightarrow} 0$ in the topology of $D([0, \infty), \mathbb{R})$.

Proof. Note that

$$
\mathrm{E}\left[\sum_{j=1}^{X_{k-1}}\left(\xi_{k, j}-1\right)\left(\eta_{k}-\lambda\right) \mid \overline{\mathcal{F}} k-1\right]=0 \quad \text { and } \sum_{k=1}^{n} \sum_{j=1}^{X_{k-1}}\left(\xi_{k, j}-1\right)\left(\eta_{k}-\lambda\right)
$$

is a martingale with respect to $\overline{\mathcal{F}}_{n}$. Then, for any $T>0$, we have

$$
\begin{gathered}
\mathrm{E}\left[\sup _{0 \leq t \leq T}\left(\frac{1}{n^{d}} \sum_{k=1}^{[n t]} \sum_{j=1}^{X_{k-1}}\left(\xi_{k, j}-1\right)\left(\eta_{k}-\lambda\right)\right)^{2}\right] \leq \frac{n^{2} \sigma^{2} \gamma^{2}}{n^{2 d}} \int_{0}^{T} \mathrm{E}\left[Y_{n}(s)\right] \mathrm{d} s, \\
\mathrm{E}\left[\sup _{0 \leq t \leq T}\left|\frac{1}{n^{d}} \sum_{k=1}^{[n t]}\left[\left(\eta_{k}-\lambda\right)^{2}-\gamma^{2}\right]\right|\right] \leq \frac{2 n \gamma^{2} T}{n^{d}} .
\end{gathered}
$$

Since $d>1$ and $\int_{0}^{t} \mathrm{E}\left[Y_{n}(s)\right] \mathrm{d} s \rightarrow \frac{1}{2} \lambda t^{2}$ as $n \rightarrow \infty$, we have Lemma 3.1. 
Now we concentrate on $V_{1, n}(\cdot)$ and $V_{2, n}(\cdot)$. For simplicity, let $\zeta_{k, j}=\xi_{k, j}-1$. Inspired by the method of Samorodnitsky et al. [15], for any fixed $\varepsilon>0$, we introduce a family of random variables $\left\{\bar{\zeta}_{k, j}: k, j=1,2, \ldots\right\}$ defined by

$$
\bar{\zeta}_{k, j}= \begin{cases}\zeta_{k, j} & \text { if }\left|\zeta_{k, j}\right| \leq a_{n} \varepsilon \\ \tilde{\zeta}_{k, j} & \text { if }\left|\zeta_{k, j}\right|>a_{n} \varepsilon\end{cases}
$$

where $\left\{\tilde{\zeta}_{k, j}: k, j=1,2, \ldots\right\}$ is the family of i.i.d. random variables with a common distribution $\mathrm{P}\left(\zeta_{k, j} \in \cdot|| \zeta_{k, j} \mid \leq a_{n} \varepsilon\right)$, and independent of $\left\{\xi_{k, j}\right\}$ and $\left\{\eta_{k}\right\}$. Set

$$
\begin{aligned}
& v_{1, n}^{\varepsilon}(k)=\sum_{j=1}^{X_{k-1}}\left(\zeta_{k, j}^{2} \mathbf{1}_{\left\{\left|\zeta_{k, j}\right|>a_{n} \varepsilon\right\}}-\mathrm{E}\left[\zeta_{k, j}^{2} \mathbf{1}_{\left\{\left|\zeta_{k, j}\right|>a_{n} \varepsilon\right\}}\right]\right), \\
& v_{2, n}^{\varepsilon}(k)=2 \sum_{j=2}^{X_{k-1}} \sum_{l=1}^{j-1}\left(\bar{\zeta}_{k, j}-\mathrm{E}\left[\bar{\zeta}_{k, j}\right]\right)\left(\bar{\zeta}_{k, l}-\mathrm{E}\left[\bar{\zeta}_{k, l}\right]\right) .
\end{aligned}
$$

Let $V_{i, n}^{\varepsilon}(t)=\sum_{k=1}^{[n t]} v_{i, n}^{\varepsilon}(k), i=1,2$. Let $Z_{n}^{\varepsilon}(t)=\left(Y_{n}(t), V_{1, n}^{\varepsilon}(t) / a_{n}^{2}, V_{2, n}^{\varepsilon}(t) / n^{3 / 2}\right)$. We first consider the weak convergence for $Z_{n}^{\varepsilon}(\cdot)$. We need the following four lemmas.

Lemma 3.2. Assume that $m=1, \gamma^{2}<\infty$, and condition (1.2) is satisfied. We have, for $t \geq 0$,

$$
\begin{gathered}
\limsup _{n \rightarrow \infty} \mathrm{E}\left[\sup _{0 \leq s \leq t} Y_{n}^{2}(s)\right] \leq\left(\lambda \sigma^{2}+2 \lambda^{2}\right) t^{2}, \\
\limsup _{n \rightarrow \infty}\left(\frac{1}{a_{n}^{2}} \mathrm{E}\left[\left|V_{1, n}^{\varepsilon}(t)\right|\right]+\frac{1}{n^{3}} \mathrm{E}\left[\left(V_{2, n}^{\varepsilon}(t)\right)^{2}\right]\right) \leq \frac{\alpha}{\alpha-2} \varepsilon^{2-\alpha} \lambda t^{2}+\left(\lambda \sigma^{6}+2 \sigma^{4} \lambda^{2}\right) t^{3} .
\end{gathered}
$$

Proof. Note that $X_{[n t]}=\sum_{k=1}^{[n t]}\left[\sum_{j=1}^{X_{k-1}} \zeta_{k, j}+\left(\eta_{k}-\lambda\right)\right]+\lambda[n t] . \quad$ By applying Doob's inequality to the martingale term we have

$$
\begin{aligned}
\mathrm{E}\left[\sup _{0 \leq s \leq t} Y_{n}^{2}(s)\right] & \leq 2 \mathrm{E}\left[\frac{1}{n^{2}} \sum_{k=1}^{[n t]}\left(\sum_{j=1}^{X_{k-1}}\left[\zeta_{k, j}+\left(\eta_{k}-\lambda\right)\right]\right)^{2}\right]+2 \lambda^{2} t^{2} \\
& \leq 2 \sigma^{2} \int_{0}^{t} \mathrm{E}\left[Y_{n}(s)\right] \mathrm{d} s+\frac{\gamma^{2} t}{n}+2 \lambda^{2} t^{2}
\end{aligned}
$$

Since $\int_{0}^{t} \mathrm{E}\left[Y_{n}(s)\right] \mathrm{d} s \rightarrow \frac{1}{2} \lambda t^{2}$ as $n \rightarrow \infty,(3.3)$ holds. We also have

$$
\begin{aligned}
\frac{1}{a_{n}^{2}} \mathrm{E}\left[\left|V_{1, n}^{\varepsilon}(t)\right|\right] & \leq \frac{1}{a_{n}^{2}} \sum_{k=1}^{[n t]} \mathrm{E}\left[\sum_{j=1}^{X_{k-1}}\left(\zeta_{k, j}^{2} \mathbf{1}_{\left\{\left|\zeta_{k, j}\right|>a_{n} \varepsilon\right\}}+\mathrm{E}\left[\zeta_{k, j}^{2} \mathbf{1}_{\left\{\left|\zeta_{k, j}\right|>a_{n} \varepsilon\right\}}\right]\right)\right] \\
& \leq \frac{2 n^{2}}{a_{n}^{2}} \mathrm{E}\left[\zeta_{1,1}^{2} \mathbf{1}_{\left\{\left|\zeta_{1,1}\right|>a_{n} \varepsilon\right\}}\right] \int_{0}^{t} \mathrm{E}\left[Y_{n}(s)\right] \mathrm{d} s \\
\frac{1}{n^{3}} \mathrm{E}\left[\left(V_{2, n}^{\varepsilon}(t)\right)^{2}\right] & =\frac{2\left(\operatorname{var} \bar{\zeta}_{1,1}\right)^{2}}{n^{3}} \sum_{k=1}^{[n t]} \mathrm{E}\left[X_{k-1}\left(X_{k-1}-1\right)\right] \\
& \leq 2 \mathrm{E}^{2}\left[\bar{\zeta}_{1,1}^{2}\right] \int_{0}^{t} \mathrm{E}\left[Y_{n}^{2}(s)\right] \mathrm{d} s
\end{aligned}
$$


By (1.2) and Karamata's theorem,

$$
\mathrm{E}\left[\zeta_{1,1}^{2} \mathbf{1}_{\left\{\left|\zeta_{1,1}\right|>a_{n} \varepsilon\right\}}\right] \sim \frac{\alpha}{\alpha-2} \frac{a_{n}^{2}}{n^{2}} \varepsilon^{2-\alpha}
$$

Note that $\mathrm{E}\left[\bar{\zeta}_{1,1}^{2}\right] \rightarrow \sigma^{2}$ and $\int_{0}^{t} \mathrm{E}\left[Y_{n}^{2}(s)\right] \mathrm{d} s \rightarrow \int_{0}^{t}\left(\frac{1}{2} \lambda \sigma^{2}+\lambda^{2}\right) s^{2} \mathrm{~d} s$ as $n \rightarrow \infty$. Then (3.4) holds.

Lemma 3.3. Under the conditions of Lemma 3.2, for fixed $\varepsilon>0$, the sequence $Z_{n}^{\varepsilon}(\cdot)$ is tight in $D\left([0, \infty), \mathbb{R}_{+} \times \mathbb{R}^{2}\right)$.

Proof. By Lemma 3.2, $\left(V_{1, n}^{\varepsilon}(t) / a_{n}^{2}, V_{2, n}^{\varepsilon}(t) / n^{3 / 2}\right)$ is a tight sequence of random vectors for every $t \geq 0$. Note that $C(t):=\limsup _{n \rightarrow \infty} \mathrm{E}\left[\sup _{0 \leq s \leq t} Y_{n}^{2}(s)\right]+1$ is a locally bounded function of $t \geq 0$. Now let $\left\{\tau_{n}\right\}$ be a sequence of stopping times bounded by $T$, and let $\left\{\delta_{n}\right\}$ be a sequence of positive constants such that $\delta_{n} \rightarrow 0$ as $n \rightarrow \infty$. We obtain, as in the calculations in (3.5) and (3.6), for sufficiently large $n$,

$$
\begin{aligned}
\frac{1}{a_{n}^{2}} \mathrm{E}\left[\left|V_{1, n}^{\varepsilon}\left(\tau_{n}+\delta_{n}\right)-V_{1, n}^{\varepsilon}\left(\tau_{n}\right)\right|\right] \leq & \frac{2 n^{2}}{a_{n}^{2}} \mathrm{E}\left[\zeta_{1,1}^{2} \mathbf{1}_{\left\{\left|\zeta_{1,1}\right|>a_{n} \varepsilon\right\}}\right] \\
& \times \int_{0}^{\left(\left[n \delta_{n}\right]+1\right) / n} \mathrm{E}\left[Y_{n}\left(\frac{\left[n \tau_{n}\right]+[n s]}{n}\right)\right] \mathrm{d} s \\
\leq & \left(\frac{2 \alpha}{\alpha-2} \varepsilon^{2-\alpha}+1\right) \int_{0}^{\delta_{n}+1 / n} C^{1 / 2}(T+s) \mathrm{d} s \\
\frac{1}{n^{3}} \mathrm{E}\left[\left(V_{2, n}^{\varepsilon}\left(\tau_{n}+\delta_{n}\right)-V_{2, n}^{\varepsilon}\left(\tau_{n}\right)\right)^{2}\right] \leq & 2\left(\sigma^{2}+1\right)^{2} \int_{0}^{\delta_{n}+1 / n} C(T+s) \mathrm{d} s .
\end{aligned}
$$

Then $\left(V_{1, n}^{\varepsilon}(\cdot) / a_{n}^{2}, V_{2, n}^{\varepsilon}(\cdot) / n^{3 / 2}\right)$ is tight in $D\left([0, \infty), \mathbb{R}^{2}\right)$ by the criterion of Aldous [1]. By Proposition 2.1, $Y_{n}(\cdot)$ is $C^{*}$-tight. Then it follows from [9, Corollary 3.33, p. 317] that $Z_{n}^{\varepsilon}(\cdot)$ is tight in $D\left([0, \infty), \mathbb{R}_{+} \times \mathbb{R}^{2}\right)$.

Lemma 3.4. Assume that the conditions of Lemma 3.2 are satisfied. Then, for fixed $\varepsilon>0$,

$$
\frac{1}{n^{3}} \sum_{k=1}^{[n t]} \mathrm{E}\left[\left(v_{2, n}^{\varepsilon}(k)\right)^{2} \mathbf{1}_{\left\{\left|v_{2, n}^{\varepsilon}(k)\right|>\epsilon n^{3 / 2}\right\}} \mid \mathcal{F}_{k-1}\right] \stackrel{\mathrm{P}}{\rightarrow} 0 \quad \text { as } n \rightarrow \infty
$$

for all $\epsilon>0$ and $t \geq 0$.

Proof. By (3.2), $\left\{\bar{\zeta}_{k, j}\right\}$ is a family of i.i.d.random variables. Fix $k$. Since $\sigma\left(\bar{\zeta}_{k, j}: j=\right.$ $1,2, \ldots)$ is independent of $\mathcal{F}_{k-1}$, we have $\mathrm{E}\left[\left|v_{2, n}^{\varepsilon}(k)\right|^{2+\delta} \mid \mathcal{F}_{k-1}\right]=2^{2+\delta} \Lambda\left(X_{k-1}\right)$ almost surely (a.s.) for $0<\delta<\alpha-2$, where

$$
\Lambda(m)=\mathrm{E}\left[\left|\sum_{j=2}^{m} \sum_{l=1}^{j-1}\left(\bar{\zeta}_{k, j}-\mathrm{E}\left[\bar{\zeta}_{k, j}\right]\right)\left(\bar{\zeta}_{k, l}-\mathrm{E}\left[\bar{\zeta}_{k, l}\right]\right)\right|^{2+\delta}\right], \quad m=2,3, \ldots
$$

Still with $k$ fixed, we note that $\sum_{l=1}^{m}\left(\bar{\zeta}_{k, l}-\mathrm{E}\left[\bar{\zeta}_{k, l}\right]\right)$ is an $\overline{\mathcal{F}}_{m}^{k}$-martingale, where

$$
\overline{\mathcal{F}}_{m}^{k}=\sigma\left(\bar{\zeta}_{k, 1}, \bar{\zeta}_{k, 2}, \ldots, \bar{\zeta}_{k, m}\right)
$$


Then, by Burkholder's inequality (see [6, p. 23]) and Minkowski's inequality,

$$
\begin{aligned}
\mathrm{E}\left[\left|\sum_{l=1}^{j-1}\left(\bar{\zeta}_{k, l}-\mathrm{E}\left[\bar{\zeta}_{k, l}\right]\right)\right|^{2+\delta}\right] & \leq C_{1} \mathrm{E}\left[\left|\sum_{l=1}^{j-1}\left(\bar{\zeta}_{k, l}-\mathrm{E}\left[\bar{\zeta}_{k, l}\right]\right)^{2}\right|^{(2+\delta) / 2}\right] \\
& \leq C_{1}\left(\sum_{l=1}^{j-1} \mathrm{E}^{2 /(2+\delta)}\left[\left|\bar{\zeta}_{k, l}-\mathrm{E}\left[\bar{\zeta}_{k, l}\right]\right|^{2+\delta}\right]\right)^{(2+\delta) / 2}
\end{aligned}
$$

where $C_{1}$ is a positive constant depending only on $\delta$. Let $\bar{\sigma}_{2+\delta}=\mathrm{E}\left[\left(\xi_{1,1}+2\right)^{2+\delta}\right]$. We have $\mathrm{E}\left[\left|\bar{\zeta}_{1,1}-\mathrm{E}\left[\bar{\zeta}_{1,1}\right]\right|^{2+\delta}\right] \leq 2 \bar{\sigma}_{2+\delta}$ for large enough $n$. Also, note that

$$
T_{k}(m)=\sum_{j=2}^{m} \sum_{l=1}^{j-1}\left(\bar{\zeta}_{k, j}-\mathrm{E}\left[\bar{\zeta}_{k, j}\right]\right)\left(\bar{\zeta}_{k, l}-\mathrm{E}\left[\bar{\zeta}_{k, l}\right]\right)
$$

is a martingale with respect to $\overline{\mathcal{F}}_{m}^{k}$ for fixed $k$. By Burkholder's inequality, Minkowski's inequality, and (3.7), we have

$$
\begin{aligned}
\Lambda(m) & \leq C_{2} \mathrm{E}\left[\left|\sum_{j=2}^{m}\left(\bar{\zeta}_{k, j}-\mathrm{E}\left[\bar{\zeta}_{k, j}\right]\right)^{2}\left(\sum_{l=1}^{j-1}\left(\bar{\zeta}_{k, l}-\mathrm{E}\left[\bar{\zeta}_{k, l}\right]\right)\right)^{2}\right|^{(2+\delta) / 2}\right] \\
& \leq C_{2}\left(\sum_{j=2}^{m} \mathrm{E}^{2 /(2+\delta)}\left[\left|\bar{\zeta}_{k, j}-\mathrm{E}\left[\bar{\zeta}_{k, j}\right]\right|^{2+\delta}\right] \mathrm{E}^{2 /(2+\delta)}\left[\left|\sum_{l=1}^{j-1}\left(\bar{\zeta}_{k, l}-\mathrm{E}\left[\bar{\zeta}_{k, l}\right]\right)\right|^{2+\delta}\right]\right)^{(2+\delta) / 2} \\
& \leq C_{1} C_{2}\left(\sum_{j=2}^{m}(j-1) \mathrm{E}^{4 /(2+\delta)}\left[\left|\bar{\zeta}_{k, j}-\mathrm{E}\left[\bar{\zeta}_{k, j}\right]\right|^{2+\delta}\right]\right)^{(2+\delta) / 2} \\
& \leq 4 C_{1} C_{2} \bar{\sigma}_{2+\delta}^{2} m^{2+\delta}
\end{aligned}
$$

where $C_{2}$ is a positive constant depending only on $\delta$. Then

$$
\frac{1}{n^{3}} \sum_{k=1}^{[n t]} \mathrm{E}\left[\left(v_{2, n}^{\varepsilon}(k)\right)^{2} \mathbf{1}_{\left\{\left|v_{2, n}^{\varepsilon}(k)\right|>\epsilon n^{3 / 2}\right\}} \mid \mathcal{F}_{k-1}\right] \leq \frac{1}{n^{\delta / 2} \epsilon^{\delta}}\left(2^{4+\delta} C_{1} C_{2} \bar{\sigma}_{2+\delta}^{2}\right) \int_{0}^{t} Y_{n}^{2+\delta}(s) \mathrm{d} s,
$$

which converges in probability to 0 by Proposition 2.1 and the continuous mapping theorem (see [2, Theorem 2.7]).

By Lemma 3.3, for any fixed $\varepsilon>0$, let $Z^{\varepsilon}(\cdot)=\left(Y^{\varepsilon}(\cdot), V_{1, \varepsilon}(\cdot), V_{2, \varepsilon}(\cdot)\right)$ be any limit point of $Z_{n}^{\varepsilon}(\cdot)$. Without loss of generality, by Skorokhod's theorem, we can assume that on some Skorokhod space $\left(\Omega^{\varepsilon}, \mathcal{F}^{\varepsilon}, \mathcal{F}_{t}^{\varepsilon}, \mathrm{P}^{\varepsilon}\right), Z_{n}^{\varepsilon}(\cdot) \stackrel{\text { a.s. }}{\longrightarrow} Z^{\varepsilon}(\cdot)$ in the topology of $D\left([0, \infty), \mathbb{R}_{+} \times \mathbb{R}^{2}\right)$.

Lemma 3.5. Assume that the conditions of Lemma 3.2 are satisfied. For any fixed $\varepsilon>0$ and $\theta=\left(\theta_{1}, \theta_{2}, \theta_{3}\right) \in \mathbb{R}^{3}$,

$$
L(t)=\mathrm{e}^{\mathrm{i}\left\langle\theta, Z^{\varepsilon}(t)\right\rangle}-1-\int_{0}^{t} \mathrm{e}^{\mathrm{i}\left\langle\theta, Z^{\varepsilon}(s)\right\rangle} A\left(Y^{\varepsilon}(s)\right) \mathrm{d} s
$$

is a complex-valued local $\mathcal{F}_{t}^{\varepsilon}$-martingale. Here $\mathrm{i}^{2}=-1,\langle\cdot, \cdot\rangle$ is the inner product of $\mathbb{R}^{3}$, and

$$
A(y)=\mathrm{i} \lambda \theta_{1}-\frac{1}{2} \sigma^{2} \theta_{1}^{2} y-\sigma^{4} \theta_{3}^{2} y^{2}+\frac{\alpha}{2} y \int_{\varepsilon^{2}}^{\infty}\left(\mathrm{e}^{\mathrm{i} \theta_{2} u}-1-\mathrm{i} \theta_{2} u\right) \frac{1}{u^{\alpha / 2+1}} \mathrm{~d} u .
$$


Proof. For any $b>1$, define the stopping times

$$
\begin{aligned}
\tau^{b} & =\inf \left\{t \geq 0:\left\|Z^{\varepsilon}(t)\right\| \geq b \text { or }\left\|Z^{\varepsilon}(t-)\right\| \geq b\right\}, \\
\tau_{n}^{b} & =\inf \left\{t \geq 0:\left\|Z_{n}^{\varepsilon}(t)\right\| \geq b \text { or }\left\|Z_{n}^{\varepsilon}(t-)\right\| \geq b\right\} .
\end{aligned}
$$

Let $Z^{b, \varepsilon}(t)=Z^{\varepsilon}\left(t \wedge \tau^{b}\right)$ and $Z_{n}^{b, \varepsilon}(t)=Z_{n}^{\varepsilon}\left(t \wedge \tau_{n}^{b}\right)$. It follows from [9, Proposition 2.11, p. 305] that, for all but countably many $b$,

$$
\tau_{n}^{b} \stackrel{\text { a.s. }}{\longrightarrow} \tau^{b} \quad \text { in } \mathbb{R} \text { and } Z_{n}^{b, \varepsilon}(\cdot) \stackrel{\text { a.s. }}{\longrightarrow} Z^{b, \varepsilon}(\cdot)
$$

in the topology of $D\left([0, \infty), \mathbb{R}_{+} \times \mathbb{R}^{2}\right)$. Define $\tau_{n}^{b}(t)=\tau_{n}^{b} \wedge t$ and $\tau^{b}(t)=\tau^{b} \wedge t$. We claim that

$$
\tau_{n}^{b}(\cdot) \stackrel{\text { a.s. }}{\longrightarrow} \tau^{b}(\cdot) \quad \text { in } C\left([0, \infty), \mathbb{R}_{+}\right) \text {as } n \rightarrow \infty .
$$

In fact, since $0 \leq \tau_{n}^{b}(t+\varepsilon)-\tau_{n}^{b}(t) \leq \varepsilon$ for any $t \geq 0$, the criterion of Aldous [1] yields tightness for $\left\{\tau_{n}^{b}(\cdot), n \geq 1\right\}$. Let $\hat{\mathcal{F}}_{k}^{n}=\sigma\left(Z_{n}^{\varepsilon}(j / n): j=0,1, \ldots, k\right)$. Note that $\left\{Z_{n}^{\varepsilon}(k / n): k \geq 1\right\}$ is a time-homogeneous Markov chain. Then

$$
\begin{aligned}
L_{n}(t)= & \mathrm{e}^{\mathrm{i}\left\langle\theta, Z_{n}^{\varepsilon}(t)\right\rangle}-1 \\
& -\sum_{k=1}^{[n t]} \mathrm{e}^{\mathrm{i}\left\langle\theta, Z_{n}^{\varepsilon}((k-1) / n)\right\rangle}\left(\mathrm{E}\left[\exp \left(\mathrm{i}\left\langle\theta, Z_{n}^{\varepsilon}\left(\frac{k}{n}\right)-Z_{n}^{\varepsilon}\left(\frac{k-1}{n}\right)\right\rangle\right) \mid \hat{\mathcal{F}}_{k-1}^{n}\right]-1\right)
\end{aligned}
$$

is a complex-valued martingale. Let $u_{n}(k)=\sum_{j=1}^{X_{k-1}}\left(\bar{\zeta}_{k, j}-\mathrm{E}\left[\bar{\zeta}_{k, j}\right]\right)$. Then

$$
\begin{aligned}
\mathrm{E}\left[\exp \left(\mathrm{i}\left\langle\theta, Z_{n}^{\varepsilon}\left(\frac{k}{n}\right)-Z_{n}^{\varepsilon}\left(\frac{k-1}{n}\right)\right\rangle\right) \mid \mathcal{F}_{k-1}^{n}\right]-1 \\
=\left(\mathrm{E}\left[\exp \left(\mathrm{i}\left(\theta_{1} \frac{u_{n}(k)}{n}+\theta_{1} \frac{\eta_{k}}{n}+\theta_{2} \frac{v_{1, n}^{\varepsilon}(k)}{a_{n}^{2}}+\theta_{3} \frac{v_{2, n}^{\varepsilon}(k)}{n^{3 / 2}}\right)\right) \mid X_{k-1}\right]-1\right) \\
+\mathrm{E}\left[\exp \left(\mathrm{i}\left(\theta_{1} \frac{u_{n}(k)}{n}+\theta_{1} \frac{\eta_{k}}{n}+\theta_{2} \frac{v_{1, n}^{\varepsilon}(k)}{a_{n}^{2}}+\theta_{3} \frac{v_{2, n}^{\varepsilon}(k)}{n^{3 / 2}}\right)\right)\right. \\
\left.\quad \times\left(\exp \left(\mathrm{i} \theta_{1}\left(\sum_{j=1}^{X_{k-1}} \frac{\zeta_{k, j}-\bar{\zeta}_{k, j}+\mathrm{E}\left[\bar{\zeta}_{k, j}\right]}{n}\right)\right)-1\right) \mid X_{k-1}\right] \\
=I_{n}(k)+J_{n}(k) .
\end{aligned}
$$

By (3.2), for fixed $k$, if $X_{k-1}$ is known then $v_{1, n}^{\varepsilon}(k), \eta_{k}$, and $\left(u_{n}(k), v_{2, n}^{\varepsilon}(k)\right)$ are independent of each other. On the other hand,

$$
\mathrm{E}\left[\theta_{1} \frac{u_{n}(k)}{n}+\theta_{3} \frac{v_{2, n}^{\varepsilon}(k)}{n^{3 / 2}} \mid X_{k-1}\right]=0 .
$$

Then

$$
\begin{aligned}
I_{n}(k)= & \mathrm{E}\left[\exp \left(\mathrm{i} \theta_{1} \frac{\eta_{k}}{n}\right)\right] \mathrm{E}\left[\exp \left(\mathrm{i} \theta_{1} \frac{u_{n}(k)}{n}+\mathrm{i} \theta_{3} \frac{v_{2, n}^{\varepsilon}(k)}{n^{3 / 2}}\right) \mid X_{k-1}\right] \\
& \times \mathrm{E}\left[\exp \left(\mathrm{i} \theta_{2} \frac{v_{1, n}^{\varepsilon}(k)}{a_{n}^{2}}\right) \mid X_{k-1}\right]-1 \\
= & I_{1, n}(k)+I_{2, n}(k),
\end{aligned}
$$


where

$$
\begin{aligned}
I_{0, n}(k)=\mathrm{E}\left[\exp \left(\mathrm{i} \theta_{1} \frac{\eta_{k}}{n}\right)\right] \mathrm{E}\left[\exp \left(\mathrm{i} \theta_{2} \frac{v_{1, n}^{\varepsilon}(k)}{a_{n}^{2}}\right) \mid X_{k-1}\right], \\
I_{1, n}(k)=I_{0, n}(k) \mathrm{E}\left[1-\frac{1}{2}\left(\theta_{1} \frac{u_{n}(k)}{n}+\theta_{3} \frac{v_{2, n}^{\varepsilon}(k)}{n^{3 / 2}}\right)^{2} \mid X_{k-1}\right]-1, \\
I_{2, n}(k)=I_{0, n}(k) \mathrm{E}\left[\exp \left(\mathrm{i} \theta_{1} \frac{u_{n}(k)}{n}+\mathrm{i} \theta_{3} \frac{v_{2, n}^{\varepsilon}(k)}{n^{3 / 2}}\right)-1\right. \\
\left.+\frac{1}{2}\left(\theta_{1} \frac{u_{n}(k)}{n}+\theta_{3} \frac{v_{2, n}^{\varepsilon}(k)}{n^{3 / 2}}\right)^{2} \mid X_{k-1}\right] .
\end{aligned}
$$

It follows from (2.4) that $n^{2} \mathrm{P}\left(\left|\zeta_{1,1}\right|>a_{n} x\right) \rightarrow x^{-\alpha}$ for $x>0$ and $\alpha>2$. Moreover, for any $K>0, n^{2} \mathrm{E}\left[\left(\zeta_{1,1} / a_{n}\right)^{2} \mathbf{1}_{\left\{\left|\zeta_{1,1}\right|>a_{n} K\right\}}\right] \rightarrow(\alpha /(\alpha-2)) K^{2-\alpha}$. Let $\mu_{n}$ be the distribution of $\zeta_{1,1} / a_{n}$. We have, as $n \rightarrow \infty$,

$$
n^{2} \int_{|u|>\varepsilon}\left(\mathrm{e}^{\mathrm{i} \theta_{2} u^{2}}-1-\mathrm{i} \theta_{2} u^{2}\right) \mu_{n}(\mathrm{~d} u) \rightarrow \alpha \int_{\varepsilon}^{\infty}\left(\mathrm{e}^{\mathrm{i} \theta_{2} u^{2}}-1-\mathrm{i} \theta_{2} u^{2}\right) \frac{1}{u^{\alpha+1}} \mathrm{~d} u .
$$

The right-hand side is equal to

$$
\frac{\alpha}{2} \int_{\varepsilon^{2}}^{\infty}\left(\mathrm{e}^{\mathrm{i} \theta_{2} u}-1-\mathrm{i} \theta_{2} u\right) \frac{1}{u^{\alpha / 2+1}} \mathrm{~d} u
$$

Let $v_{n}$ be the distribution of $\eta_{1} / n$. We have

$$
\begin{aligned}
I_{0, n}(k)= & \mathrm{E}\left[\exp \left(\mathrm{i} \theta_{1} \frac{\eta_{k}}{n}\right)\right]\left(\mathrm{E}\left[\exp \left(\mathrm{i} \theta_{2}\left(\frac{\zeta_{1,1}}{a_{n}}\right)^{2} \mathbf{1}_{\left\{\left|\zeta_{1,1}\right|>a_{n} \varepsilon\right\}}\right)\right]\right)^{X_{k-1}} \\
& \times \exp \left(-\mathrm{i} \theta_{2} X_{k-1} \mathrm{E}\left[\left(\frac{\zeta_{1,1}}{a_{n}}\right)^{2} \mathbf{1}_{\left\{\left|\zeta_{1,1}\right|>a_{n} \varepsilon\right\}}\right]\right) \\
= & \exp \left(\mathrm{i} \theta_{1} \frac{\lambda}{n}+X_{k-1} \int_{|u|>\varepsilon}\left(\mathrm{e}^{\mathrm{i} \theta_{2} u^{2}}-1-\mathrm{i} \theta_{2} u^{2}\right) \mu_{n}(\mathrm{~d} u)+X_{k-1} \varrho_{1, n}+\varrho_{2, n}\right),
\end{aligned}
$$

where

$$
\begin{aligned}
& \varrho_{1, n}=\sum_{j=2}^{\infty} \frac{(-1)^{j-1}}{j}\left(\int_{|u|>\varepsilon}\left(\mathrm{e}^{\mathrm{i} \theta_{2} u^{2}}-1\right) \mu_{n}(\mathrm{~d} u)\right)^{j} \\
& \varrho_{2, n}=\int\left(\mathrm{e}^{\mathrm{i} \theta_{1} u}-1-\mathrm{i} \theta_{1} u\right) v_{n}(\mathrm{~d} u)+\sum_{j=2}^{\infty} \frac{(-1)^{j-1}}{j}\left(\int\left(\mathrm{e}^{\mathrm{i} \theta_{1} u}-1\right) v_{n}(\mathrm{~d} u)\right)^{j} .
\end{aligned}
$$

Note that $n^{2}\left|\varrho_{1, n}\right| \leq n^{2}\left(\int_{|u|>\varepsilon}\left(\mathrm{e}^{\mathrm{i} \theta_{2} u^{2}}-1\right) \mu_{n}(\mathrm{~d} u)\right)^{2} \rightarrow 0$ and $\left|\varrho_{2, n}\right| \leq \theta_{1}^{2}\left(2 \lambda^{2}+\gamma^{2}\right) / n^{2}$. Also,

$$
\begin{aligned}
& \mathrm{E}\left[\frac{1}{2}\left(\theta_{1} \frac{u_{n}(k)}{n}+\theta_{3} \frac{v_{2, n}^{\varepsilon}(k)}{n^{3 / 2}}\right)^{2} \mid X_{k-1}\right] \\
& =\frac{\theta_{1}^{2}}{2 n^{2}} \operatorname{var}\left(\bar{\zeta}_{1,1}\right) X_{k-1}+\frac{\theta_{3}^{2}}{n^{3}}\left(\operatorname{var}\left(\bar{\zeta}_{1,1}\right)\right)^{2}\left(X_{k-1}^{2}-X_{k-1}\right)
\end{aligned}
$$


By (3.10), (3.11), and (3.12), if $X_{k-1} / n \leq b$ then $I_{1, n}(k)=A_{n}\left(X_{k-1} / n\right)$, where

$$
\begin{aligned}
A_{n}(y)= & \mathrm{i} \frac{\theta_{1} \lambda}{n}-\frac{\theta_{1}^{2}}{2 n} \operatorname{var}\left(\bar{\zeta}_{1,1}\right) y-\frac{\theta_{3}^{2}}{n}\left(\operatorname{var}\left(\bar{\zeta}_{1,1}\right)\right)^{2} y\left(y-\frac{1}{n}\right) \\
& +n y \int_{|u|>\varepsilon}\left(\mathrm{e}^{\mathrm{i} \theta_{2} u^{2}}-1-\mathrm{i} \theta_{2} u^{2}\right) \mu_{n}(\mathrm{~d} u)+\kappa_{n}(y)
\end{aligned}
$$

for $0 \leq y \leq b$. Here $\kappa_{n}(y)$ is some complex-valued function satisfying $\sup _{0 \leq y \leq b}\left|\kappa_{n}(y)\right| \leq$ $C_{b} o(1 / n)$ for some constant $C_{b}$ depending on $b$. It is not hard to show that $n \bar{A}_{n}(y) \rightarrow A(y)$ uniformly on $y \in[0, b]$ for fixed $\theta$. Recall that $Y_{n}^{b}(t)=Y_{n}\left(t \wedge \tau_{n}^{b}\right)$. By (3.8) and [5, Problem 26, p. 153], we obtain

$$
\int_{0}^{t} \exp \left(\mathrm{i}\left\langle\theta, Z_{n}^{\varepsilon, b}(t-)\right\rangle\right) n A_{n}\left(Y_{n}^{b}(s)\right) \mathrm{d} s \stackrel{\text { a.s. }}{\longrightarrow} \int_{0}^{t} \exp \left(\mathrm{i}\left\langle\theta, Z^{\varepsilon, b}(s)\right\rangle\right) A\left(Y^{b}(s)\right) \mathrm{d} s
$$

in the topology of $C([0, \infty), \mathbb{C})$. Note that $[n t] / n \rightarrow t$ in $C\left([0, \infty), \mathbb{R}_{+}\right)$. By (3.9), $[5$, Problem 13, p. 151], and [9, Proposition 1.23], we have

$$
\int_{0}^{\left[n\left(t \wedge \tau_{n}^{b}\right)\right] / n} \exp \left(\mathrm{i}\left\langle\theta, Z_{n}^{\varepsilon, b}(t)\right\rangle\right) n A_{n}\left(Y_{n}^{b}(s)\right) \mathrm{d} s \stackrel{\text { a.s. }}{\longrightarrow} \int_{0}^{t \wedge \tau^{b}} \exp \left(\mathrm{i}\left\langle\theta, Z^{\varepsilon, b}(s)\right\rangle\right) A\left(Y^{b}(s)\right) \mathrm{d} s
$$

in the topology of $C([0, \infty), \mathbb{C})$. For any $\epsilon>0$,

$$
\begin{aligned}
\left|I_{2, n}(k)\right| \leq & \frac{\epsilon}{6}\left(\frac{\theta_{1}^{2}}{n^{2}} \operatorname{var}\left(\bar{\zeta}_{1,1}\right) X_{k-1}+\frac{2 \theta_{3}^{2}}{n^{3}}\left(\operatorname{var}\left(\bar{\zeta}_{1,1}\right)\right)^{2}\left(X_{k-1}^{2}-X_{k-1}\right)\right) \\
& +\frac{4 \theta_{1}^{2}}{n^{2}} \mathrm{E}\left[\left(u_{n}(k)\right)^{2} \mathbf{1}_{\left\{\left|\theta_{1} u_{n}(k)\right|>\epsilon n / 2\right\}}\right]+\frac{4 \theta_{3}^{2}}{n^{3}} \mathrm{E}\left[\left(v_{2}^{\varepsilon}(k)\right)^{2} \mathbf{1}_{\left\{\left|\theta_{3} v_{2}^{\varepsilon}(k)\right|>\epsilon n^{3 / 2} / 2\right\}}\right] .
\end{aligned}
$$

Without loss of generality, assume that $\theta_{1}, \theta_{3}>0$. As in the proof of Lemma 3.4, we have, for $0<\delta<\alpha-2$,

$$
\mathrm{E}\left[\left|u_{n}(k)\right|^{2+\delta}\right] \leq 2 C \bar{\sigma}_{2+\delta} X_{k-1}^{(2+\delta) / 2},
$$

where $C$ is a constant depending on $\delta$. Since $\epsilon$ is arbitrary, it follows from (3.14) and Lemma 3.4 that

$$
\sum_{k=1}^{\left[n\left(t \wedge \tau_{n}^{b}\right)\right]} \exp \left(\mathrm{i}\left\langle\theta, Z_{n}^{\varepsilon}\left(\frac{k-1}{n}\right)\right\rangle\right) I_{2, n}(k) \stackrel{\mathrm{P}}{\rightarrow} 0
$$

in $C([0, \infty), \mathbb{C})$. For large enough $n$,

$$
\begin{gathered}
\left|J_{n}(k)\right| \leq \frac{X_{k-1}}{n}\left|\theta_{1}\right|\left(\mathrm{E}\left[\left|\zeta_{1,1}\right| \mathbf{1}_{\left\{\left|\zeta_{1,1}\right|>a_{n} \varepsilon\right\}}\right]+2 \mathrm{E}\left[\left|\zeta_{1,1}\right|\right] \mathrm{P}\left(\left|\zeta_{1,1}\right|>a_{n} \varepsilon\right)\right. \\
\left.+2\left|\mathrm{E}\left[\zeta_{1,1} \mathbf{1}_{\left\{\left|\zeta_{1,1}\right| \leq a_{n} \varepsilon\right\}}\right]\right|\right)
\end{gathered}
$$

Note that $E\left[\zeta_{1,1}\right]=0$, so we have

$$
\left|\mathrm{E}\left[\zeta_{1,1} \mathbf{1}_{\left\{\left|\zeta_{1,1}\right| \leq a_{n} \varepsilon\right\}}\right]\right|=\left|\mathrm{E}\left[\zeta_{1,1} \mathbf{1}_{\left\{\left|\zeta_{1,1}\right|>a_{n} \varepsilon\right\}}\right]\right| \leq \mathrm{E}\left[\left|\zeta_{1,1}\right| \mathbf{1}_{\left\{\left|\zeta_{1,1}\right|>a_{n} \varepsilon\right\}}\right] \sim \frac{\alpha}{(\alpha-1) \varepsilon^{\alpha-1}} \frac{a_{n}}{n^{2}}
$$

and $a_{n} / n \rightarrow 0$ for $\alpha>2$. Then

$$
\sum_{k=1}^{\left[n\left(t \wedge \tau_{n}^{b}\right)\right]} \exp \left(\mathrm{i}\left\langle\theta, Z_{n}^{\varepsilon}\left(\frac{k-1}{n}\right)\right\rangle\right) J_{n}(k) \stackrel{\text { a.s. }}{\longrightarrow} 0 \quad \text { in } C([0, \infty), \mathbb{C}) .
$$


Thus, we have $L_{n}\left(t \wedge \tau_{n}^{b}\right) \stackrel{\text { a.s. }}{\longrightarrow} L\left(t \wedge \tau^{b}\right)$ in $D([0, \infty), \mathbb{C})$ (passing to a subsequence if necessary). For almost all $t \geq 0, L_{n}\left(t \wedge \tau_{n}^{b}\right) \stackrel{\text { a.s. }}{\longrightarrow} L\left(t \wedge \tau^{b}\right)$ in $\mathbb{C}$. Fix $T>0$ arbitrarily. It is not hard to see that there exists a constant $K$ such that $\left|L_{n}\left(t \wedge \tau_{n}^{b}\right)\right| \leq K$ for large enough $n$ and any $t \leq T$. Then, for almost all $t \leq T, L_{n}\left(t \wedge \tau_{n}^{b}\right) \stackrel{L_{1}}{\rightarrow} L\left(t \wedge \tau^{b}\right)$ as $n \rightarrow \infty$. Since $L\left(t \wedge \tau^{b}\right)$ is right continuous and bounded for $t \leq T, L\left(t \wedge \tau^{b}\right)$ is a martingale. Note that $\tau^{b} \rightarrow \infty$ as $b \rightarrow \infty$, so $L(t)$ is a local martingale.

Proposition 3.1. Assume that the conditions of Lemma 3.2 are satisfied. For any fixed $\varepsilon>0$, $Z_{n}^{\varepsilon}(\cdot)$ converges in distribution on $D\left([0, \infty), \mathbb{R}_{+} \times \mathbb{R}^{2}\right)$ to the process $Z^{\varepsilon}(\cdot)$ defined by

$$
\begin{gathered}
Y^{\varepsilon}(t)=\lambda t+\int_{0}^{t} \sigma \sqrt{Y^{\varepsilon}(s)} \mathrm{d} W^{\varepsilon}(s), \quad V_{2, \varepsilon}(t)=\int_{0}^{t} \sqrt{2} \sigma^{2} Y^{\varepsilon}(s) \mathrm{d} B^{\varepsilon}(s), \\
V_{1, \varepsilon}(t)=\int_{0}^{t} \int_{\varepsilon^{2}}^{\infty} \int_{0}^{Y^{\varepsilon}(s)} u \tilde{N}^{\varepsilon}(\mathrm{d} s, \mathrm{~d} u, \mathrm{~d} \varsigma),
\end{gathered}
$$

where $W^{\varepsilon}(t)$ and $B^{\varepsilon}(t)$ are Brownian motions, and $N^{\varepsilon}(\mathrm{d} s, \mathrm{~d} u, \mathrm{~d} \varsigma)$ is a Poisson random measure on $(0, \infty) \times \mathbb{R}_{+} \times(0, \infty)$ with intensity $(\alpha / 2) \mathrm{d} s u^{-\alpha / 2-1} \mathrm{~d} u \mathrm{~d} \zeta$. Here $W^{\varepsilon}, B^{\varepsilon}$, and $N^{\varepsilon}$ are independent of each other and $\tilde{N}^{\varepsilon}(\mathrm{d} s, \mathrm{~d} u, \mathrm{~d} \varsigma)=N^{\varepsilon}(\mathrm{d} s, \mathrm{~d} u, \mathrm{~d} \varsigma)-(\alpha / 2) \mathrm{d} s u^{-\alpha / 2-1} \mathrm{~d} u \mathrm{~d} \varsigma$.

Proof. It follows from Lemma 3.5 and [9, Theorem 2.42, p. 86] that $Z^{\varepsilon}(\cdot)$ is a semimartingale and it admits the canonical representation

$$
Y^{\varepsilon}(t)=\lambda t+Y_{c}^{\varepsilon}(t), \quad V_{2, \varepsilon}=V_{2, \varepsilon}^{c}, \quad V_{1, \varepsilon}=\int_{0}^{t} \int_{\mathbb{R}_{+}} u \tilde{J}^{\varepsilon}(\mathrm{d} s, \mathrm{~d} u),
$$

with $\left(Y_{c}^{\varepsilon}(t), V_{2, \varepsilon}^{c}(t)\right)$ a vector of two continuous local martingales with quadratic covariation process $\left(\int_{0}^{t} c_{i j}(s) \mathrm{d} s\right)_{i, j=1}^{2}$, where $c_{11}(s)=\sigma^{2} Y^{\varepsilon}(s), c_{12}(s) \equiv 0$, and $c_{22}(s)=2 \sigma^{4}\left(Y^{\varepsilon}(s)\right)^{2}$, and $J^{\varepsilon}(\mathrm{d} t, \mathrm{~d} u)$ is an integer-valued random measure on $(0, \infty) \times \mathbb{R}_{+}$with compensator $\hat{J}^{\varepsilon}(\mathrm{d} t, \mathrm{~d} u)=(\alpha / 2) Y^{\varepsilon}(t) \mathrm{d} t \mathbf{1}_{\left(\varepsilon^{2}, \infty\right)}(u) u^{-\alpha / 2-1} \mathrm{~d} u$. Let $\rho(\mathrm{d} u, \mathrm{~d} \varsigma)=(\alpha / 2) u^{-\alpha / 2-1} \mathrm{~d} u \mathrm{~d} \varsigma$. Since $(\alpha / 2) u^{-\alpha / 2-1} \mathrm{~d} u$ is supported by $(0, \infty)$, we can check that, for any $(a, b) \subset(0, \infty)$,

$$
\frac{\alpha}{2} Y^{\varepsilon}(t) \int_{a \wedge \varepsilon^{2}}^{b \wedge \varepsilon^{2}} u^{-\alpha / 2-1} \mathrm{~d} u=\rho(\{u: \tilde{\theta}(t, u, \varsigma) \in(a, b)\}),
$$

where $\tilde{\theta}(t, u, \varsigma)=u \mathbf{1}_{\left(\varepsilon^{2}, \infty\right)}(u) \mathbf{1}_{\left(0, Y^{\varepsilon}(t)\right]}(\varsigma)$. By Ikeda and Watanabe [8, pp. 84 and 93], there exists a standard extension of the original probability space supporting two independent Brownian motions $W^{\varepsilon}(\mathrm{t})$ and $B^{\varepsilon}(t)$ and a Poisson random measure $N^{\varepsilon}(\mathrm{d} t, \mathrm{~d} u, \mathrm{~d} \varsigma)$ on $(0, \infty) \times$ $\mathbb{R}_{+} \times(0, \infty)$ with intensity $\mathrm{d} t \rho(\mathrm{d} u, \mathrm{~d} \varsigma)$ such that (3.15) holds, and, for any $(a, b) \subset(0, \infty)$,

$$
J^{\varepsilon}((0, t] \times(a, b))=\int_{0}^{t} \int_{\mathbb{R}_{+} \times(0, \infty)} \mathbf{1}_{(a, b)}(\tilde{\theta}(s, u, \varsigma)) N^{\varepsilon}(\mathrm{d} s, \mathrm{~d} u, \mathrm{~d} \varsigma) .
$$

Then (3.16) holds. Thus, $Z^{\varepsilon}(\cdot)$ is the solution of the stochastic equation system (3.15) and (3.16). The pathwise uniqueness of the solution for the above equation system is obvious (see [4]). Also, by Lemma 3.3 we have the weak convergence for $Z_{n}^{\varepsilon}(\cdot)$.

Let $Z_{n}(t)=\left(Y_{n}(t), V_{1, n}(t) / a_{n}^{2}, V_{2, n}(t) / n^{3 / 2}\right)$. We have the following proposition.

Proposition 3.2. Assume that the conditions of Lemma 3.2 are satisfied with $2<\alpha<4$. Let $W(t)$ and $B(t)$ be Brownian motions, and let $N(\mathrm{~d} s, \mathrm{~d} u, \mathrm{~d} \varsigma)$ be a Poisson random measure 
$(0, \infty) \times \mathbb{R}_{+} \times(0, \infty)$ with intensity $(\alpha / 2) \mathrm{d} s u^{-\alpha / 2-1} \mathrm{~d} u \mathrm{~d} \varsigma$. Suppose that $W, B$, and $N$ are independent of each other. Then $Z_{n}(\cdot)$ converges in distribution on $D\left([0, \infty), \mathbb{R}_{+} \times \mathbb{R}^{2}\right)$ to the process $Z(\cdot)$, which can be defined by

$$
\begin{gathered}
Y(t)=\lambda t+\int_{0}^{t} \sigma \sqrt{Y(s)} \mathrm{d} W(s), \quad V_{2}(t)=\int_{0}^{t} \sqrt{2} \sigma^{2} Y(s) \mathrm{d} B(s), \\
V_{1}(t)=\int_{0}^{t} \int_{0}^{\infty} \int_{0}^{Y(s)} u \tilde{N}(\mathrm{~d} s, \mathrm{~d} u, \mathrm{~d} \varsigma),
\end{gathered}
$$

where $\tilde{N}(\mathrm{~d} s, \mathrm{~d} u)=N(\mathrm{~d} s, \mathrm{~d} u, \mathrm{~d} \varsigma)-(\alpha / 2) \mathrm{d} s u^{-\alpha / 2-1} \mathrm{~d} u \mathrm{~d} \zeta$.

Proof. Obviously, there exists a unique solution for the equation system (3.17)-(3.18), denoted by $Z(\cdot)$. Let $Z^{\varepsilon}(\cdot)$ be defined by (3.15)-(3.16). It is not hard to see that $Z^{\varepsilon}(\cdot)$ converges in distribution on $D\left([0, \infty), \mathbb{R}_{+} \times \mathbb{R}^{2}\right)$ to the process $Z(\cdot)$ as $\varepsilon \rightarrow 0$. We claim that, for any $T>0$ and $r>0$,

$$
\lim _{\varepsilon \rightarrow 0} \limsup _{n \rightarrow \infty} \mathrm{P}\left(\sup _{0 \leq t \leq T}\left|Z_{n}^{\varepsilon}(t)-Z_{n}(t)\right|>r\right)=0 .
$$

Recall that $\overline{\mathcal{F}}_{n}=\sigma\left(X_{0}, \xi_{k, j}, \eta_{k}: 1 \leq k \leq n, j \geq 1\right)$. Then

$$
V_{1, n}(t)-V_{1, n}^{\varepsilon}(t)=\sum_{k=1}^{[n t]} \sum_{j=1}^{X_{k-1}}\left(\zeta_{k, j}^{2} \mathbf{1}_{\left\{\left|\zeta_{k, j}\right| \leq a_{n} \varepsilon\right\}}-\mathrm{E}\left[\zeta_{k, j}^{2} \mathbf{1}_{\left\{\left|\zeta_{k, j}\right| \leq a_{n} \varepsilon\right\}}\right]\right)
$$

is an $\overline{\mathcal{F}}_{[n t]}$-martingale. By Doob's inequality,

$$
\begin{aligned}
\limsup _{n \rightarrow \infty} \frac{1}{a_{n}^{4}} \mathrm{E}\left[\sup _{0 \leq t \leq T}\left(V_{1, n}(t)-V_{1, n}^{\varepsilon}(t)\right)^{2}\right] & \leq \limsup _{n \rightarrow \infty} \frac{4}{a_{n}^{4}} \sum_{k=1}^{[n T]} \mathrm{E}\left[X_{k-1}\right] \mathrm{E}\left[\zeta_{1,1}^{4} \mathbf{1}_{\left\{\left|\zeta_{1,1}\right| \leq a_{n} \varepsilon\right\}}\right] \\
& \leq \frac{4 \alpha}{4-\alpha} \varepsilon^{4-\alpha} \int_{0}^{T} \mathrm{E}[Y(s)] \mathrm{d} s .
\end{aligned}
$$

Since $2<\alpha<4, \lim _{\varepsilon \rightarrow 0} \lim \sup _{n \rightarrow \infty} \mathrm{E}\left[\sup _{0 \leq t \leq T}\left(V_{1, n}(t)-V_{1, n}^{\varepsilon}(t)\right)^{2}\right] / a_{n}^{4}=0$. Note that

$$
\begin{aligned}
V_{2, n}(t)-V_{2, n}^{\varepsilon}(t)= & 2 \sum_{k=1}^{[n t]} \sum_{i \neq j}^{X_{k-1}}\left(\zeta_{k, i} \mathbf{1}_{\left\{\left|\zeta_{k, i}\right|>a_{n} \varepsilon\right\}}-\mathrm{E}\left[\zeta_{k, i} \mathbf{1}_{\left\{\left|\zeta_{k, i}\right|>a_{n} \varepsilon\right\}}\right]\right) \zeta_{k, j} \mathbf{1}_{\left\{\left|\zeta_{k, j}\right| \leq a_{n} \varepsilon\right\}} \\
& -2 \sum_{k=1}^{[n t]} \sum_{i \neq j}^{X_{k-1}}\left(\tilde{\zeta}_{k, i} \mathbf{1}_{\left\{\left|\zeta_{k, i}\right|>a_{n} \varepsilon\right\}}-\mathrm{E}\left[\tilde{\zeta}_{k, i}\right] \mathrm{P}\left(\left|\zeta_{k, i}\right|>a_{n} \varepsilon\right)\right) \zeta_{k, j} \mathbf{1}_{\left\{\left|\zeta_{k, j}\right| \leq a_{n} \varepsilon\right\}} \\
& -2 \sum_{k=1}^{[n t]} \sum_{i<j}^{X_{k-1}}\left(\tilde{\zeta}_{k, i} \mathbf{1}_{\left\{\left|\zeta_{k, i}\right|>a_{n} \varepsilon\right\}}-\mathrm{E}\left[\bar{\zeta}_{k, i}\right]\right)\left(\tilde{\zeta}_{k, j} \mathbf{1}_{\left\{\left|\zeta_{k, j}\right|>a_{n} \varepsilon\right\}}-\mathrm{E}\left[\bar{\zeta}_{k, j}\right]\right) \\
& +2 \sum_{k=1}^{[n t]} \sum_{i<j}^{X_{k-1}} \zeta_{k, i} \mathbf{1}_{\left\{\left|\zeta_{k, i}\right|>a_{n} \varepsilon\right\}} \zeta_{k, j} \mathbf{1}_{\left\{\left|\zeta_{k, j}\right|>a_{n} \varepsilon\right\}} \\
= & \sum_{k=1}^{4} J_{k, n}(t) .
\end{aligned}
$$


Note that $J_{1, n}(k / n) / n^{3 / 2}$ is an $\overline{\mathcal{F}}_{k}$-martingale. For any $t \geq 0$,

$$
\begin{aligned}
& \frac{1}{n^{3}} \sum_{k=1}^{[n t]} \mathrm{E}\left[\left(\sum_{i \neq j}^{X_{k-1}}\left(\zeta_{k, i} \mathbf{1}_{\left\{\left|\zeta_{k, i}\right|>a_{n} \varepsilon\right\}}-\mathrm{E}\left[\zeta_{k, i} \mathbf{1}_{\left\{\left|\zeta_{k, i}\right|>a_{n} \varepsilon\right\}}\right]\right) \zeta_{k, j} \mathbf{1}_{\left\{\left|\zeta_{k, j}\right|>a_{n} \varepsilon\right\}}\right)^{2} \mid \overline{\mathcal{F}}_{k-1}\right] \\
& \quad \leq \frac{a_{n}^{2}}{n^{2}} \frac{\alpha}{(\alpha-2) \varepsilon^{\alpha-2}} \mathrm{E}\left[\zeta_{1,1}^{2}\right] \int_{0}^{t} Y_{n}^{2}(s) \mathrm{d} s+\frac{a_{n}^{4}}{n^{5}} \frac{\alpha^{3}}{(\alpha-1)^{2}(\alpha-2) \varepsilon^{3 \alpha-4}} \int_{0}^{t} Y_{n}^{3}(s) \mathrm{d} s
\end{aligned}
$$

which converges in probability to 0 by Lemma 2.1 and $\alpha>2$. It follows from the martingale central limit theorem that $J_{1, n}(t) / n^{3 / 2} \stackrel{\mathrm{P}}{\rightarrow} 0$ in the topology of $D([0, \infty), \mathbb{R})$. For $J_{3, n}(t) / n^{3 / 2}$,

$$
\frac{1}{n^{3 / 2}} \mathrm{E}\left[\sup _{0 \leq t \leq T}\left|J_{3, n}(t)\right|\right] \leq 8\left(\mathrm{E}\left[\left|\zeta_{1,1}\right|\right] \frac{1}{n^{5 / 4} \varepsilon^{\alpha}}+\frac{\alpha}{\alpha-1} \frac{a_{n}}{n^{5 / 4} \varepsilon^{\alpha-1}}\right)^{2} \int_{0}^{T} \mathrm{E}\left[Y_{n}^{2}(s)\right] \mathrm{d} s,
$$

which converges to 0 by $\alpha>2$. Similarly, $J_{2}(t) / n^{3 / 2}$ and $J_{4}(t) / n^{3 / 2}$ have the same results as $J_{1, n}(t) / n^{3 / 2}$ and $J_{3, n}(t) / n^{3 / 2}$, respectively. By (3.21) and (3.20), (3.19) holds. Proposition 3.2 follows from Proposition 3.1 and [2, Theorem 3.2].

Let $N_{1}(\mathrm{~d} s, \mathrm{~d} u)$ be a Poisson random measure on $(0, \infty) \times \mathbb{R}_{+}$with intensity

$$
\frac{\alpha}{2} \mathrm{~d} s u^{-\alpha / 2-1} \mathrm{~d} u
$$

independent of $W, B$, and $N$. Define

$$
\begin{aligned}
S_{\alpha / 2}(t)= & \int_{0}^{t} \int_{0}^{\infty} \int_{0}^{Y(s)}(Y(s))^{-2 / \alpha} \mathbf{1}_{\{Y(s) \neq 0\}} u \tilde{N}(\mathrm{~d} s, \mathrm{~d} u, \mathrm{~d} \varsigma) \\
& +\int_{0}^{t} \int_{0}^{\infty} u \mathbf{1}_{\{Y(s)=0\}} \tilde{N}_{1}(\mathrm{~d} s, \mathrm{~d} u) .
\end{aligned}
$$

Then $S_{\alpha / 2}(t)$ is a martingale. By Itô's formula, it is not hard to see that $S_{\alpha / 2}(t)$ is a one-sided $(\alpha / 2)$-stable process with exponent defined by (2.5). Thus, we also have

$$
V_{1}(t)=\int_{0}^{t} Y^{2 / \alpha}(s) \mathrm{d} S_{\alpha / 2}(s) .
$$

Proof of Theorem 2.1. For case (i), note that $n^{3 / 2} / a_{n}^{2} \rightarrow 0$. Write $V_{n}(t) / a_{n}^{2}=V_{1, n}(t) / a_{n}^{2}+$ $\left(n^{3 / 2} / a_{n}^{2}\right)\left(V_{2, n}(t) / n^{3 / 2}\right)+V_{3, n}(t) / a_{n}^{2}$. By Lemma 3.1, Proposition 3.2, (3.22), and the continuous mapping theorem, the weak convergence result holds with (2.6). In a similar way, we also have cases (ii)-(iii) when $\alpha<4$. Now we concentrate on case (iii) when $\alpha \geq 4$. As in the proofs of Lemmas 3.2-3.5 and Proposition 3.1, we can prove that $\left(Y_{n}(\cdot), V_{2, n}(\cdot) / n^{3 / 2}\right)$ converges in distribution on $D\left([0, \infty), \mathbb{R}_{+} \times \mathbb{R}\right)$ to the process $\left(Y(\cdot), V_{2}(\cdot)\right)$ defined by (3.17). If $\alpha>4, \mathrm{E}\left[\xi_{1,1}^{4}\right]<\infty$ and

$$
\frac{1}{n^{3}} \sum_{k=1}^{[n t]} \mathrm{E}\left[\left(\sum_{j=1}^{X_{k-1}}\left(\zeta_{k, j}^{2}-\sigma^{2}\right)\right)^{2} \mid \overline{\mathcal{F}}_{k-1}\right] \leq \frac{1}{n} \mathrm{E}\left[\zeta_{1,1}^{4}\right] \int_{0}^{t} Y_{n}(s) \mathrm{d} s
$$


which converges in probability to 0 for any $t \geq 0$. By the martingale central limit theorem (see [6, p. 58]), $V_{1, n}(t) / n^{3 / 2} \stackrel{\mathrm{p}}{\rightarrow} 0$ in the topology of $D([0, \infty), \mathbb{R})$. If $\alpha=4$, we note that

$$
\begin{aligned}
\frac{1}{n^{3 / 2}} \mathrm{E}\left[\sup _{0 \leq s \leq t}\left|V_{1, n}(s)\right|\right] \leq & \frac{2}{n^{3 / 2}} \mathrm{E}^{1 / 2}\left[\left(\sum_{k=1}^{[n t]} \sum_{j=1}^{X_{k-1}}\left(\zeta_{k, j}^{2} \mathbf{1}_{\left\{\left|\zeta_{k, j}\right| \leq a_{n}\right\}}-\mathrm{E}\left[\zeta_{k, j}^{2} \mathbf{1}_{\left\{\left|\zeta_{k, j}\right| \leq a_{n}\right\}}\right]\right)\right)^{2}\right] \\
& +\frac{2}{n^{3 / 2}} \sum_{k=1}^{[n t]} \sum_{j=1}^{X_{k-1}} \mathrm{E}\left[\zeta_{k, j}^{2} \mathbf{1}_{\left\{\left|\zeta_{k, j}\right|>a_{n}\right\}}\right] \\
\leq & \left.\frac{4}{n^{3}} \sum_{k=1}^{[n t]} \mathrm{E}\left[X_{k-1}\right] \mathrm{E}\left[\zeta_{1,1}^{4} \mathbf{1}_{\left\{\left|\zeta_{1,1}\right| \leq a_{n}\right\}}\right]\right)^{1 / 2} \\
& +\frac{2}{n^{3 / 2}} \sum_{k=1}^{[n t]} \mathrm{E}\left[X_{k-1}\right] \mathrm{E}\left[\zeta_{1,1}^{2} \mathbf{1}_{\left\{\left|\zeta_{1,1}\right|>a_{n}\right\}}\right] .
\end{aligned}
$$

Since $\alpha=4$, it follows from Karamata's theorem (see [3, Proposition 1.5.9]) that

$$
\mathrm{E}\left[\zeta_{1,1}^{2} \mathbf{1}_{\left\{\left|\zeta_{1,1}\right|>a_{n}\right\}}\right] \sim \frac{2 a_{n}^{2}}{n^{2}}
$$

and that $\mathrm{E}\left[\zeta_{1,1}^{4} \mathbf{1}_{\left\{\left|\zeta_{1,1}\right| \leq a_{n}\right\}}\right]=\tilde{L}\left(a_{n}\right)-a_{n}^{4} \mathrm{P}\left(\left|\zeta_{1,1}\right|>a_{n}\right)$ for some positive function $\tilde{L}(x)$ slowly varying at $\infty$. In this case, $a_{n}^{2} / n^{3 / 2} \rightarrow 0$ and $\tilde{L}\left(a_{n}\right) / n \rightarrow 0$. Then $V_{1, n}(t) / n^{3 / 2} \stackrel{\mathrm{P}}{\rightarrow} 0$ in the topology of $D([0, \infty), \mathbb{R})$. Thus, by Lemma 3.1, Theorem 2.1 follows from the continuous mapping theorem.

Based on Proposition 2.1, Wei and Winnicki [17] gave the asymptotic properties of the CLSE $\left(\hat{m}_{n}, \hat{\lambda}_{n}\right)$ of $(m, \lambda)$ as follows.

Lemma 3.6. ([17].) If $m=1, \sigma^{2}<\infty$, and $\gamma^{2}<\infty$, then

$$
\left(\begin{array}{c}
n\left(\hat{m}_{n}-m\right) \\
\hat{\lambda}_{n}-\lambda
\end{array}\right) \stackrel{\mathrm{D}}{\rightarrow}\left(\begin{array}{c}
\frac{Y^{2}(1) / 2-\left(Y(1)+\sigma^{2} / 2\right) \int_{0}^{1} Y(t) \mathrm{d} t}{\int_{0}^{1} Y^{2}(t) \mathrm{d} t-\left(\int_{0}^{1} Y(t) \mathrm{d} t\right)^{2}} \\
\frac{\int_{0}^{1} \sigma Y^{1 / 2}(t) \mathrm{d} W(t) \int_{0}^{1} Y^{2}(t) \mathrm{d} t-\int_{0}^{1} Y(t) \mathrm{d} t \int_{0}^{1} \sigma Y^{3 / 2}(t) \mathrm{d} W(t)}{\int_{0}^{1} Y^{2}(t) \mathrm{d} t-\left(\int_{0}^{1} Y(t) \mathrm{d} t\right)^{2}}
\end{array}\right)
$$

where $Y(\cdot)$ and $W(\cdot)$ are given in (2.3).

Proof of Theorem 2.2. Write $V_{n}(t)=M_{n}(t)+H_{n}(t)$, where

$$
\begin{aligned}
M_{n}(t)= & \sum_{k=1}^{[n t]} \sum_{j=1}^{X_{k-1}}\left(\zeta_{k, j}^{2} \mathbf{1}_{\left\{\left|\zeta_{k, j}\right| \leq a_{n}\right\}}-\mathrm{E}\left[\zeta_{k, j}^{2} \mathbf{1}_{\left\{\left|\zeta_{k, j}\right| \leq a_{n}\right\}}\right]\right)+2 \sum_{k=1}^{[n t]} \sum_{j=2}^{X_{k-1}} \sum_{l=1}^{j-1} \zeta_{k, j} \zeta_{k, l} \\
& +2 \sum_{k=1}^{[n t]} \sum_{j=1}^{X_{k-1}} \zeta_{k, j}\left(\eta_{k}-\lambda\right), \\
H_{n}(t)= & \sum_{k=1}^{[n t]} \sum_{j=1}^{X_{k-1}}\left(\zeta_{k, j}^{2} \mathbf{1}_{\left\{\left|\zeta_{k, j}\right|>a_{n}\right\}}-\mathrm{E}\left[\zeta_{k, j}^{2} \mathbf{1}_{\left\{\left|\zeta_{k, j}\right|>a_{n}\right\}}\right\}\right)+\sum_{k=1}^{[n t]}\left(\left(\eta_{k}-\lambda\right)^{2}-\gamma^{2}\right) .
\end{aligned}
$$


Now we turn to case (i). In this case, $M_{n}(t)$ is an $\overline{\mathcal{F}}_{[n t]}$-martingale, and, for any $t \geq 0$,

$$
\limsup _{n \rightarrow \infty} \frac{1}{a_{n}^{4}} \mathrm{E}\left[M_{n}^{2}(t)\right] \leq \frac{\alpha}{4-\alpha} \int_{0}^{t} \mathrm{E}[Y(s)] \mathrm{d} s .
$$

Here $H_{n}(t)$ has paths of finite variation on bounded intervals. Denote its finite variation by $\int_{0}^{t}\left|\mathrm{~d} H_{n}(s)\right|$. We have

$$
\limsup _{n \rightarrow \infty} \frac{1}{a_{n}^{2}} \mathrm{E}\left[\int_{0}^{t}\left|\mathrm{~d} H_{n}(s)\right|\right] \leq \frac{2 \alpha}{\alpha-2} \int_{0}^{t} \mathrm{E}[Y(s)] \mathrm{d} s .
$$

Then, by Theorem 2.1 and [12, Theorem 2.2],

$$
\left(Y_{n}(t), \frac{V_{n}(t)}{a_{n}^{2}}, \frac{1}{a_{n}^{2}} \int_{0}^{t} Y_{n}(s) \mathrm{d} V_{n}(s)\right) \rightarrow\left(Y(t), V(t), \int_{0}^{t} Y(s) \mathrm{d} V(s)\right)
$$

in distribution on $D\left([0, \infty), \mathbb{R}_{+} \times \mathbb{R}^{2}\right)$, where $V(\cdot)$ is defined by (2.6). Here $V(t)$ and $\int_{0}^{t} Y(s) \mathrm{d} V(s)$ are stochastically continuous. Also, note that

$$
\begin{aligned}
& \frac{n^{2}}{a_{n}^{2}}\left(\bar{\sigma}_{n}^{2}-\sigma^{2}\right)=\frac{\left(1 / a_{n}^{2}\right) \int_{0}^{t} Y_{n}(s) \mathrm{d} V_{n}(s)-\left(V_{n}(1) / a_{n}^{2}\right) \int_{0}^{t} Y_{n}(s) \mathrm{d} s}{\int_{0}^{1} Y_{n}^{2}(s)-\left(\int_{0}^{1} Y_{n}(s) \mathrm{d} s\right)^{2}}, \\
& \frac{n}{a_{n}^{2}}\left(\bar{\gamma}_{n}^{2}-\gamma^{2}\right)=\frac{V_{n}(1)}{a_{n}^{2}}-\frac{n^{2}}{a_{n}^{2}}\left(\bar{\sigma}_{n}^{2}-\sigma^{2}\right) \int_{0}^{t} Y_{n}(s) \mathrm{d} s .
\end{aligned}
$$

By (3.24) and the continuous mapping theorem, we have (2.9) for case (i). Cases (ii)-(iii) can be proved in a similar way. On the other hand,

$$
\begin{aligned}
\hat{U}_{k}^{2}-U_{k}^{2}= & \left(\hat{m}_{n}-m\right)^{2} X_{k-1}^{2}+\left(\hat{\lambda}_{n}-\lambda\right)^{2}+2\left(\hat{m}_{n}-m\right)\left(\hat{\lambda}_{n}-\lambda\right) X_{k-1} \\
& -2\left(\hat{m}_{n}-m\right) U_{k} X_{k-1}-2\left(\hat{\lambda}_{n}-\lambda\right) U_{k} .
\end{aligned}
$$

As in the above proof, again by Lemma 3.6 and [18, Lemma 2.7], we can show that the above results also hold for $\bar{\sigma}_{n}^{2}$ and $\bar{\gamma}_{n}^{2}$.

\section{Acknowledgement}

We thank an anonymous referee for pointing out some mistakes in the manuscript.

\section{References}

[1] Aldous, D. (1978). Stopping times and tightness. Ann. Prob. 6, 335-340.

[2] Billingsley, P. (1999). Convergence of Probability Measures. John Wiley, New York.

[3] Bingham, N. H., Goldie, C. M. And Teugels, J. L. (1987). Regular Variation. Cambridge University Press.

[4] Dawson, D. A. And Li, Z. H. (2006). Skew convolution semigroups and affine Markov processes. Ann. Prob. 34, 1103-1142.

[5] Ethier, S. N. And Kurtz, T. G. (1986). Markov Processes. John Wiley, New York.

[6] Hall, P. and Heyde, C. (1980). Martingale Limit Theory and Its Applications. Academic Press, New York.

[7] Heyde, C. C. (1974). On estimating the variance of the offspring distribution in a simple branching process. Adv. Appl. Prob. 6, 421-433.

[8] Ikeda, N. And Watanabe, S. (1989). Stochastic Differential Equations and Diffusion Processes (North-Holland Math. Library 24), 2nd edn. North-Holland, Amsterdam.

[9] JACod, J. And Shiryaev, A. N. (1987). Limit Theorems for Stochastic Processes. Springer, Berlin. 
[10] Kawazu, K. and Watanabe, S. (1971). Branching processes with immigration and related limit theorems. Theory Prob. Appl. 16, 36-54.

[11] Klimko, L. A. And Nelson, P. I. (1978). On conditional least squares estimation for stochastic processes. Ann. Statist. 6, 629-642.

[12] Kurtz, T. G. And Protter, P. (1991). Weak limit theorems for stochastic integrals and stochastic differential equations. Ann. Prob. 19, 1035-1070.

[13] LI, Z. (2005). A limit theorem for discrete Galton-Watson branching processes with immigration. J. Appl. Prob. 43, 289-295.

[14] Samorodnitsky, G. And Taqqu, M. S. (1994). Stable Non-Gaussian Random Processes. Chapman and Hall, New York.

[15] Samorodnitsky, G., Rachev, S. T., Kurz-Kim, J. R. and Stoyanov, S. V. (2007). Asymptotic distribution of unbiased linear estimators in the presence of heavy-tailed stochastic regressors and residuals. Prob. Math. Statist. 27, 275-302.

[16] WeI, C. Z. AND WinNiCKI, J. (1989). Some asymptotic results for the branching process with immigration. Stochastic Process. Appl. 31, 261-282.

[17] WeI, C. Z. AND WinNiCKI, J. (1990). Estimation of the means in the branching process with immigration. Ann. Statist. 18, 1757-1773.

[18] WinNiCKI, J. (1991). Estimation of the variances in the branching process with immigration. Prob. Theory Relat. Fields 88, 77-106. 